\title{
How the Narlikar Argument of Quantum Gravity Can Be Combined with the Cosmological Constant We Calculated to Obtain Quantum Gravity Effects for Plank Length Values, as Opposed to 2 Times Planck Length Values
}

\author{
Andrew Beckwith \\ Physics Department, Chongqing University, Huxi Campus, Chongqing, China \\ Email: rwill9955b@gmai.com
}

How to cite this paper: Beckwith, A. (2021) How the Narlikar Argument of Quantum Gravity Can Be Combined with the Cosmological Constant We Calculated to Obtain Quantum Gravity Effects for Plank Length Values, as Opposed to 2 Times Planck Length Values. Journal of High Energy Physics, Gravitation and Cosmology, 7, 445-451.

https://doi.org/10.4236/jhepgc.2021.72025

Received: February 23, 2021

Accepted: April 3, 2021

Published: April 6, 2021

Copyright $\odot 2021$ by author(s) and Scientific Research Publishing Inc. This work is licensed under the Creative Commons Attribution International License (CC BY 4.0).

http://creativecommons.org/licenses/by/4.0/

\begin{abstract}
We take the results where we reduplicate the Book "Dark Energy" by M. Li, $\mathrm{X}-\mathrm{D}$. Li, and Y. Wang, zero-point energy calculation, as folded in with the Klauder methodology, as given in a prior publication. From there we first access the Rosen solution to a mini universe energy to ascertain an energy value of $t$, the pre-inflationary near singularity, then access what would be needed as to inject information into our universe. We then close with an argument by Narilkar as to a quantum bound on the Einstein-Hilbert action integral, so as to obtain quantum Gravity. Narlikar omits the cosmological constant. We keep it in, for our overall conclusion about the cosmological constant and its relevance to Quantum gravity.
\end{abstract}

\section{Keywords}

Minimum Scale Factor, Cosmological Constant, Space-Time Bubble, Bouncing Cosmologies

\section{Introduction-How We Can Link a Cosmological Constant to Quantum Effects}

What we are doing is to take the results of [1] and [2] reduplicated and using the Rosen [3] calculation to obtain a minimum energy which would be needed to move past the near singularity in order to obtain a linkage to the computed 
cosmological constant and quantum gravity, adopting an argument by Narlikar [4]. What Narlikar does is to bound the Einstein-Hilbert action integral for a black hole via Planck's h bar constant, to obtain $L \leq 2 \ell_{\text {Planck }}$ i.e., stating that quantum effects are important at lengths twice the Plank length. We will in the end duplicate this idea but with the cosmological constant included which we claim leads to $L \leq \ell_{\text {Planck }}$, i.e., the cosmological constant makes the introduction of quantum gravity commensurate with Planck length. In addition we also specify arguments as to holographic limits [5], and a near quantum bounce [6] as to ascertain what conditions would be needed to have information outside a near singularity condition of space-time we think is congruent to a start to the universe for a quantum system commensurate to a black hole for the onset of information needed for inflation [5] [7]. That also involves using [8] methodologies as to the linkage between geometric optics and quantum mechanics for our system [8].

\section{Methods}

Our methodologies specifically refer to the formalism built up in reference [1] as given in [1] and [2] which give both the $\mathrm{Li}, \mathrm{Li}$, and Wang solution to the cosmological constant and then from there obtain the redo of the [4] calculation with an eye toward linking our cosmological constant to the onset of quantum effects in a non singular start point for expansion of the universe.

\subsection{We Compare Two Types of Cosmological Constant Values Given in [1]}

$$
\begin{aligned}
& \Lambda \approx \frac{-\left[\frac{V_{0}}{3 \gamma-1}+2 \tilde{N}+\frac{\gamma(3 \gamma-1)}{8 \pi G t_{\text {Planck }}}\right]}{\frac{1}{\kappa} \int \sqrt{-g} \mathrm{~d}^{3} x}+c_{1} \frac{16 \pi}{M_{P}^{2}} \cdot\left(\rho-\frac{\rho^{2}}{2|\sigma|}\right) \\
& \Leftrightarrow \rho_{D E}=\left.\left.\frac{\Lambda}{8 \pi G} \Leftrightarrow \Lambda \approx \hbar \cdot 8 \pi G \cdot \frac{(2 \pi)^{4}}{\lambda_{D E}^{4}}\right|_{\lambda_{D E} \approx 10^{30} \rho_{\text {Planck }}} \equiv 8 \pi \cdot \frac{(2 \pi)^{4}}{\lambda_{D E}^{4}}\right|_{\lambda_{D E} \approx 10^{30}}
\end{aligned}
$$

To compare these two values we can state that within the bubble, that just before the bubble boundary, we have $\tilde{N}=0$, i.e. we pick $c_{1}$ so that the two are equivalent in value, with $\sigma$ a surface tension of the bubble of space-time, just before cosmological expansion [1] [6].

Hence, we also would be looking at the following equation.

$$
\begin{aligned}
\Lambda & \approx \gamma \cdot t^{2}+8 \pi V_{0} \cdot\left\{\sqrt{\frac{8 \pi V_{0}}{\gamma(3 \gamma-1)}} \cdot t\right\}^{\sqrt{\frac{\gamma}{4 \pi}}}-\sqrt{\frac{8 \pi}{\gamma}} \mid \\
& \approx \frac{-\left[\frac{V_{0}}{3 \gamma-1}+\frac{\gamma(3 \gamma-1)}{8 \pi}\right]}{\frac{1}{\kappa} \int \sqrt{-g} \mathrm{~d}^{3} x}+c_{1} \frac{16 \pi}{M_{P}^{2}} \cdot\left(\rho-\frac{\rho^{2}}{2|\sigma|}\right)
\end{aligned}
$$




$$
\left.\approx \hbar \cdot 8 \pi G \cdot \frac{(2 \pi)^{4}}{\lambda_{D E}^{4}}\right|_{\lambda_{D E} \approx 10^{30} \ell_{\text {Planck }}}=\left.8 \pi \cdot \frac{(2 \pi)^{4}}{\lambda_{D E}^{4}}\right|_{\lambda_{D E} \approx 10^{30}}
$$

In Equation (1) we will also make the following identification $\left.\rho \approx \rho_{D E} \approx \hbar \cdot \frac{(2 \pi)^{4}}{\lambda_{D E}^{4}}\right|_{\lambda_{D E} \approx 10^{30}}$, i.e., just before the bubble, we will factor in a very large bubble tension, $\sigma$ [6]. Doing so would be to have an optimal $c_{1}$ value, which we use to obtain quantum effects in early space-time.

\subsection{What We Obtain If We Model the Cosmological Constant This Way?}

We do not confine ourselves to Isotropic backgrounds. We also do share with Rosen [4] a procedure as to rendition a linkage between classical to quantum mechanical treatment of our problem, but our work is more in tune with using what Powell and Craseman in their book on quantum mechanics used, [8] which is like a limiting case of geometric optics.

Hence this expanded treatment whereas the Rosen treatment as Corda outlined is the most effective for quantum black holes is used as a shortcut to analyze a pre big bang to a big bang transformation of space-time.

\subsection{Final Reference to High Frequency Gravitational Waves}

From [9], Maggiore, we have

$$
\begin{aligned}
& \left(1+z_{\text {initial era }}\right) \equiv \frac{a_{\text {today }}}{a_{\text {initial era }}} \approx\left(\frac{\omega_{\text {Earth orbit }}}{\omega_{\text {initia era }}}\right)^{-1} \\
& \Rightarrow\left(1+z_{\text {initial era }}\right) \omega_{\text {Earth orbit }} \approx 10^{25} \omega_{\text {Earth orbit }} \approx \omega_{\text {initial era }}
\end{aligned}
$$

where $\quad c($ light speed $) \approx \omega_{\text {initial era }} \cdot\left(\lambda_{\text {initial post buble }}=\ell_{\text {Planck }}\right)$ and $\quad \Delta E \approx \hbar \omega_{\text {initial era }}$ and that dimensional comparison with having a temperature built up so as $\Delta E \approx \hbar \omega_{\text {initial era }}$ where $T_{\text {universe }} \approx T_{\text {Plank temerature }}=1.22 \times 10^{19} \mathrm{GeV}$. If so, then the Planck era temperature would be extremely high leading to a change in temperature from the Pre Planckian conditions to Planck era leading to, if $d$ is the dimension of space-time

$$
\Delta E=\frac{d(\operatorname{dim})}{2} \cdot k_{B} \cdot T_{\text {universe }}
$$

In doing so, be assuming

$$
\omega_{\text {initial era }} \approx \frac{C}{\ell_{\text {planck }}} \leq 1.8549 \times 10^{43} \mathrm{~Hz}
$$

where we would be assuming so then we would be looking at frequencies on Earth from gravitons of mass $m$ (graviton) less than or equal to

$$
\omega_{\text {Earth orbit }} \leq 10^{-25} \omega_{\text {initial era }}
$$

And this partly due to the transference of cosmological "information" as given in [10] for a phantom bounce type of construction. Further point that since we 
have that gravitons travel at nearly the speed of light [9], that gravitons are formed from the surface of a bubble of space-time up to the electroweak era that mass values of the order of $10^{-65}$ grams (rest mass of relic gravitons) would increase due to extremely high velocity would lead to enormous $\Delta E \approx \hbar \omega_{\text {initial era }}$ values per graviton, which would make the conflation of ultrahigh temperatures with gravitons traveling at nearly the speed of light as given in Equation (5) as compared with $\Delta E \approx \hbar \omega_{\text {initial era }}$

We can compare this with the Rosen [3] value of energy for a mini universe of (from a Schrodinger equation) with ground state mass of $m=\sqrt{\pi} M_{\text {Planck }}$ and an energy of

$$
E_{\hat{n}}=\frac{-G m^{5}}{2 \pi^{2} \hbar^{2} \widehat{n}^{2}}
$$

Our preliminary supposition is that Equation (7) could represent the initial energy of a Pre Planckian Universe and that Equation (4) be the thermal energy dumped in due to the use of Cyclic Conformal cosmology (maybe in multiverse form) so that if there is a buildup of energy greater than the magnitude of Equation (7) due to thermal buildup of temperature due to a continual intake of matter-energy, we have a release of Gravitons in great number which would commence as a domain wall broke down about in the Planckian era with a temperature of the magnitude of Planck Energy information number of. And this ties in with release of information $N$ (information) for which we have a total Graviton mass of

$$
M_{\text {graviton total }}=n_{\text {graviton }} \cdot m_{\text {graviton }}
$$

where we will be looking at a value of "information" of initially

$$
\mathbb{N}(\text { inf })=\frac{9 \pi}{\ln 2} \cdot\left(\frac{n_{\text {graviton }} \cdot m_{\text {graviton }}}{m_{\text {planck }}}\right)^{2} \approx \frac{9 \pi \cdot\left(n_{\text {graviton }}\right)^{2}}{\ln 2} \cdot 10^{-120}
$$

Now use the following approximation of the Universe, initially having the entropy of a black hole, i.e., we are using Ng Infinite Quantum statistics, [11] [12]

$$
S_{\text {Universe }} \propto S_{B H} \cong \frac{A(\text { area })}{4 \cdot \ell_{\text {Planck }}^{2}} \approx \frac{9 n_{Q}}{4} \approx n_{\text {graviton }}
$$

In taking this step, we are making use of [3] having the following radius used, namely using in our model of a black hole, the quantum "atom "approximation.

$$
r\left(n_{Q}\right) \approx \frac{3 \sqrt{n_{Q}} \cdot \ell_{\text {Planck }}}{2 \sqrt{\pi}}
$$

In order to have non vanishing information according to [7] we would need to specify having

$$
\mathbb{N}(\text { inf })=\frac{9 \pi}{\ln 2} \cdot\left(\frac{n_{\text {graviton }} \cdot m_{\text {graviton }}}{m_{\text {planck }}}\right)^{2} \approx \frac{729 \pi \cdot\left(n_{Q}\right)^{2}}{16 \cdot \ln 2} \cdot 10^{-120}
$$

Given this, if 


$$
\begin{aligned}
& \mathbb{N}(\text { inf })=\frac{9 \pi}{\ln 2} \cdot\left(\frac{n_{\text {graviton }} \cdot m_{\text {graviton }}}{m_{\text {planck }}}\right)^{2} \approx \frac{729 \pi \cdot\left(n_{Q}\right)^{2}}{16 \cdot \ln 2} \cdot 10^{-120} \geq 1 \\
& \Rightarrow E_{\hat{n}=n_{Q}}=\frac{-G \cdot\left(m=\sqrt{\pi n_{Q}} \cdot m_{\text {planck }}\right)^{5}}{2 \pi^{2} \hbar^{2} n_{Q}^{2}} \approx \frac{-G \cdot\left(\sqrt{\pi n_{Q}} \cdot\left(m_{\text {planck }}\right)^{5}\right)}{2 \hbar^{2}} \\
& \underset{\hbar=m_{\text {planck }}=G=1}{\longrightarrow} E_{\hat{n}=n_{Q}} \simeq \frac{-\left(\sqrt{\pi n_{Q}}\right)}{2} \approx \text { large negative value }
\end{aligned}
$$

We speculate that in order to have a large negative binding energy that this will mean we have then an enormous initial thermal energy from a multiverse cyclic conformal cosmology input as to undo this so as to initiate a new cycle of creation, i.e. breaking the binding energy would require Planck level temerature values. And this is a topic which will be researched furthermore in great detail. And the necessary thermal heat would drive having enormously high initial frequencies.

\subsection{And Now Our Conclusing Words as to a Quantization Limit to Pursue, If the Early Universe Has Characteristics of a Pre Planckian Black Hole}

In order to do this, we adapt an argument used by [4] as using the quantization of an action which we write using [4] first in the case of no cosmological constant, namely if $\mathfrak{I}$ is an action integral with the form of the Einstein-Hilbert least action of which: $L$ is a radius, and

$$
\begin{aligned}
\delta \mathfrak{I} & =0 \\
\mathfrak{I} & \leq \hbar
\end{aligned}
$$

Equation (15) is an imposed upon quantization limit where we use from [4]

$$
\mathfrak{I}=\left.\frac{c^{4}}{16 \pi G} \int \Re \sqrt{-g} \mathrm{~d}^{4} x \approx \frac{2 \pi^{4} \rho L^{4} c}{3}\right|_{t=L / c} \leq \hbar
$$

In case of using a black hole limit and constant energy density $\rho$, [4] argues for $L$

$$
\text { Quantization near singularity if } L \leq 2 \ell_{\text {Planck }}
$$

In the case of when the comological constant is NOT zero we impose

$$
\mathfrak{J}=\left.\frac{c^{4}}{16 \pi G} \int(\Re-2 \Lambda) \cdot \sqrt{-g} \mathrm{~d}^{4} x \approx \frac{2 \pi^{4} \rho L^{4} c}{3}\right|_{t=L / c}-\frac{2 L^{4}}{c} \cdot \Lambda \leq \hbar
$$

Here, $\rho$ is an energy density and in the case of no cosmological constant we would use

$$
\rho L^{2} \leq\left(3 c^{2} / 8 \pi G\right) \text { if } \Lambda=0
$$

And

$$
\mathfrak{I}=\left.\frac{c^{4}}{16 \pi G} \int \Re \cdot \sqrt{-g} \mathrm{~d}^{4} x \approx \frac{2 \pi^{4} \rho L^{4} c}{3}\right|_{t=L / c} \leq L^{2} c^{3} / 4 G
$$


We argue as does [4] that when there is no cosmological costant that Equation (19) and Equation (15) hold we are obtaining Equation (20) so that

$$
L \leq 2 \ell_{\text {Planck }} \text { as a quantum length limit. }
$$

In the case where we use Equation (18) instead of Equation (19) we would instead see quantization

$$
L \leq \ell_{\text {Planck }} \text { as a quantum limit }
$$

This final set of arguments if the early universe had the characteristics of a Black hole will be pursued in future research. This in tandem for obtaining an optimal understanding of the quantum characteristics embodied in [5], [7] with [5] holographic information

$$
\mathbb{N}(\text { inf })=\frac{9 \pi}{\ln 2} \cdot\left(\frac{n_{\text {graviton }} \cdot m_{\text {graviton }}}{m_{\text {planck }}}\right)^{2} \approx \frac{729 \pi \cdot\left(n_{Q}\right)^{2}}{16 \cdot \ln 2} \cdot 10^{-120} \geq 1
$$

As well as picking $c_{1}$ to give a quantum interpretation of Equation (1) and Equation (2). This would allow for an investigation of ideas in [7] [8] [13] [14] and [15]. Finally the brillaint pioneering work of John Klauder deserves its well deserved accolades, which made the first equation possible, as can be referenced in [16].

\section{Acknowledgements}

This work is supported in part by National Nature Science Foundation of China grant No. 11375279.

\section{Conflicts of Interest}

The author declares no conflicts of interest regarding the publication of this paper.

\section{References}

[1] Beckwith, A. (2021) A Solution of the Cosmological Constant, Using Multiverse Version of Penrose CCC Cosmology, and Enhanced Quantization Compared. https://doi.org/10.20944/preprints202102.0208.v1

[2] Li, M., Li, X.-D., Wang, S. and Wang, Y. (2015) Dark Energy. Peking University Press, World Scientific, Singapore.

[3] Rosen, N. (1993) Quantum Mechanics of a Miniuniverse. International Journal of Theoretical Physics, 32, 1435-1440. https://doi.org/10.1007/BF00675204

[4] Narlinkar, J.V. (1982) Quantum Fluctuations near the Classical Space-Time Singularity. Proceedings of the Workshop on Gravitation and Relativistic Astrophysics, Ahmedabhad, 18-20 January 1982, 135-151.

[5] Haranas, I. and Gkigkitzis, I. (2014) The Mass of Graviton and Its Relation to the Number of Information According to the Holographic Principle. International Scholarly Research Notices, 2014, Article ID: 718251. https://doi.org/10.1155/2014/718251

[6] Freeze, K., Brown, M. and Kinney, W. (2012) The Phantom Bounce, a New Propos- 
al for an Oscillating Cosmology. In: Mercini-Houghton, L. and Vaas, R., Eds., The Arrows of Time, a Debate in Cosmology, Fundamental Theories in Physics, Volume 172, Springer Verlag, Heidelberg, 149-156.

https://doi.org/10.1007/978-3-642-23259-6 7

[7] Novello, M. and Neves, R.P. (2003) The Mass of the Graviton and the Cosmological Constant. Classical and Quantum Gravity, 20, L67-L73.

https://doi.org/10.1088/0264-9381/20/6/101

[8] Powell, J.L. and Crasemann, B. (1961) Quantum Mechanics. Addison and Wesley Company Inc., Reading.

[9] Maggiorie, M. (2008) Gravitational Waves, Volume 1, Theory and Experiment. Oxford University Press, New York.

[10] Wang, Q.D., Zhu, Z. and Unruh, W.G. (2017) How the Huge Energy of Quantum Vacuum Gravitates to Drive the Slow Accelerating Expansion of the Universe. Physical Review D, 95, Article ID: 103504. https://doi.org/10.1103/PhysRevD.95.103504

[11] Ng, Y.J. (2008) Spacetime Foam from Entropy and Holography to Infinite Statistics and Nonlocality. Entropy, 10, 441-461. https://doi.org/10.3390/e10040441

[12] Ng, Y.J. (2007) Holographic Foam, Dark Energy and Infinite Statistics. Physics Letters $B, 657,10-14$. https://arxiv.org/abs/gr-qc/0703096 https://doi.org/10.1016/j.physletb.2007.09.052

[13] Sola, J. (2013) Cosmological Constant and Vacuum Energy: Old and New Ideas. Journal of Physics. Conference Series, 453, Article ID: 012015.

https://arxiv.org/abs/1306.1527 https://doi.org/10.1088/1742-6596/453/1/012015

[14] Seminar, P., Duplantier, B. and Rivasseau, V. (2003) Poincaré Seminar 2002: Vacuum Energy-Renormalization. Progress in Mathematical Physics, Vol. 30. Birkhäuser Verlag, Basel. https://doi.org/10.1007/978-3-0348-8075-6

[15] Kieffer, K. (2012) Quantum Gravity. 3rd Edition, Oxford Science Publications, Oxford University Press, Oxford.

[16] Klauder, J.R. (2015) Enhanced Quantization: Particles, Fields \& Gravity. World Scientific, Singapore. https://doi.org/10.1142/9452 